\title{
Possible Modulatory Role of ARC Gene Variants in Mood Disorders
}

\author{
Concetta Crisafulli ${ }^{1 *}$, Marco Calabrò ${ }^{1, *}$, Laura Mandelli ${ }^{2}$, Sheng-Min Wang ${ }^{3}$, Soo-Jung Lee ${ }^{3}$, Changsu Han ${ }^{4}$, \\ Ashwin Patkar ${ }^{5}$, Prakash Masand ${ }^{6}$, Chi-Un Pae ${ }^{3,7}$, Daniel Souery ${ }^{8}$, Julien Mendlewicz ${ }^{9}$, Alessandro Serretti ${ }^{2}$ \\ ${ }^{1}$ Department of Biomedical and Dental Sciences and Morphofunctional Images, University of Messina, Messina, ${ }^{2}$ Department of Biomedical \\ and NeuroMotor Sciences, University of Bologna, Bologna, Italy, ${ }^{3}$ Department of Psychiatry, College of Medicine, The Catholic University of \\ Korea, ${ }^{4}$ Department of Psychiatry, Korea University College of Medicine, Seoul, Korea, ${ }^{5}$ Department of Psychiatry and Behavioural Sciences, \\ Rush University Medical Center, Chicago, IL, ${ }^{6}$ Global Medical Education, New York, NY, USA, ${ }^{7}$ Cell Death Disease Research Center, College \\ of Medicine, The Catholic University of Korea, Seoul, Korea, ${ }^{8}$ Centre Européen de Psychologie Medicale, Laboratoire de Psychologie Medicale, \\ Universitè Libre de Bruxelles and Psy Pluriel, ${ }^{9}$ Department of Psychiatry, Universitè Libre de Bruxelles, Brussels, Belgium
}

Objective: The genetic background of mood disorders is gradually emerging through the use of large multicenter samples but a detailed phenotyping is complementary in elucidating the role of modulating variants.

Methods: In the present paper we focused on the possible modulatory effects of ARC gene variants on two independent mood disorder samples of European ( $n=246$ bipolar disorder) and Korean ( $n=132$ bipolar disorder; $n=242$ major depressive disorder [MDD]) ancestry.

Results: No result survived Bonferroni correction, however we evidenced promising trend toward possible association between ARC gene variants and mood disorder phenotypes. In particular, we evidenced weak correlations of ARC single nucleotide polymorphisms with depressive symptoms severity (evaluated through Hamilton depression rating scale scores) in the MDD Korean (rs7465272) and European (rs11167152) samples. Additionally rs10110456 was found to be related to Family History, while rs7465272 was related to suicide risk in the Korean sample. Finally, rs7465272 was associated with body mass index in the European sample.

Conclusion: Overall, ARC gene variants may have a partial role in modulatory effect on treatment efficacy or phenotypes of mood disorders. Further studies, on larger samples may provide a better understanding on the role of ARC gene variants in the symptom severity and treatment outcomes in patients with mood disorders.

KEY WORDS: ARC gene; Depressive disorder; Bipolar disorder; Mood disorder.

\section{INTRODUCTION}

Mood disorders have a large societal burden, therefore are the focus of healthcare research since their direct and indirect costs [1]. The main concern around mood disorders is the lack of a definite treatment. The actual drug therapy frequently fails to show a complete efficacy; partial responders and non-responders are, in fact, commonly observed in clinical practice [2]. The main reason explaining this partial efficacy ultimately lie in the complex

Received: June 20, 2019 / Accepted: July 29, 2019

Address for correspondence: Chi-Un Pae

Department of Psychiatry, Bucheon St. Mary's Hospital, College

of Medicine, The Catholic University of Korea, 327 Sosa-ro,

Wonmi-gu, Bucheon 14647, Korea

E-mail: pae@catholic.ac.kr

ORCID: https://orcid.org/0000-0002-6774-9417

*These authors contributed equally to this study. background behind mood disorders. Both environmental and genetic factors concur to drugs' efficacy inter-individual variation. In particular, the biological background is an important research focus since it may provide new targets for drugs development and biomarkers to preemptive test drugs' efficacy [3].

Many investigations were performed with the aim of identifying the biological processes involved in the development of mood disorders [4-8]. Despite the years of research, this field remain pretty active and provided many promising candidate genes for further testing and profiling. In fact, most recent studies on very large samples evidence a number of modulating gene variants [9]. This fact is a normal occurrence given the polygenetic nature of mood disorders and the consequential low effect of each variant toward the phenotype.

(c) This is an Open-Access article distributed under the terms of the Creative Commons Attribution Non-Commercial License (http://creativecommons.org/licenses/by-nc/4.0) which permits unrestricted non-commercial use, distribution, and reproduction in any medium, provided the original work is properly cited. 
Among the genes showing an interesting link with psychiatric disorders there is ARC (activity regulated cytoskeleton associated protein) [10-12]. In particular, its potential association with Mood Disorders and antidepressant efficacy was already hinted in a previous work by our group [13]. ARC is an early onset gene that encodes for a protein likely to be involved in the regulation of the actin cytoskeleton [14,15] and microtubules [16]. ARC was demonstrated to be essential for the maintenance of dendritic spines density and morphology $[17,18]$ and seems to be significantly involved in mood disorders mechanics. In particular, it was hypothesized that some drugs may elicit their effects through the control on ARC expression, and the consequent modulation of neuroplasticity and neurotransmission processes [12].

In this paper we focused on ARC and in particular on the role of 4 single nucleotide polymorphisms (SNPs), namely rs10110456, rs11167152, rs7465272, rs10097505, on treatment efficacy In this paper our aim was to further contribute to the literature regarding $A R C$ role in mood disorders, in particular we evaluated the same SNPs we investigated in a precedent work [13] in two samples of moderate size and of different ethnicity. At the same time, we attempted to find possible similarities between two genetically distinct populations.

\section{METHODS}

\section{Samples}

\section{European (EU) sample}

Two-hundreds and forty-six (246) patients affected by Bipolar Disorder were recruited in the 'Psy Pluriel' center, Centre European de Psychologie Médicale and the Department of Psychiatry of Erasme Hospital in Brussels. A detailed description of the sample has been reported elsewhere [19]. In brief, the Clinical Outcome Measures for Bipolar Disorder (COPE-BD) project enrolled patients that met the Diagnostic and Statistical Manual of Mental Disorders 4th edition (DSM-IV) criteria for a diagnosis of Bipolar type I/II disorders. A structured examination tool was used to assess socio-demographic characteristics, psychiatric antecedents, diagnosis, current and previous treatments of subjects. Lifetime and current diagnosis, course of illness and comorbidities were assessed through the Mini-International Neuropsychiatric Interview (MINI) [20].

\section{Korean (KOR) sample}

One-hundred and thirty-two (132) patients diagnosed with BD and two-hundreds and forty-two (242) patients diagnosed with major depressive disorder (MDD) for a total of 374 subjects were recruited in the Department of Psychiatry of Seoul St. Mary's Hospital. The diagnosis were performed according to DSM-IV criteria [20] for bipolar disorder and major depressive disorder, respectively. For this sample, three-hundred and twenty-six (326) controls were also available at the same site. Controls were recruited among hospital staff and non-psychiatric hospital patients, who did not satisfied criteria for current or past psychiatric disorder. Recruitment details and exclusion criteria were previously reported $[21,22]$. All patients had to be eligible for pharmacological treatment. Patients and controls were evaluated for psychiatric disorders by MINI [20]. Other characteristics of included subjects were collected through a structured interview and review of clinical charts.

The local ethical committees approved the study procedures, and all the subjects were included after they had signed an informed consent (approval no. HC10TISI0031 and OM021).

\section{Main Evaluations}

Response to antidepressant treatment, remission and resistance to treatments (primary outcomes) were defined according to the previous research [23]. Briefly, response to treatment is defined as a $\geq 50 \%$ improvement of HDRS scores from baseline to endpoint; remission as a HDRS score of $\leq 7$ at the endpoint; resistance as non-response to at least two adequate consecutive antidepressant trials (including the present) [23]. All subjects were treated in a naturalistic setting.

\section{Selection of Polymorphisms and Genotyping}

The following criteria were applied to select polymorphisms: 1) reported prevalence of at least $5 \%$ for the variant allele among Caucasians (data from http://hapmap. ncbi.nlm.nih.gov/); 2) previous evidence of possible modulation effect. Among eligible polymorphisms, the choice was performed taking into account previous findings in literature. The list of genotyped polymorphisms is shown in Supplementary Table 2 (available online). SNPs showing genotyping rate $<80 \%$ were included in the analyses, however the obtained results were considered as sugges- 
tive only.

Genomic DNA was purified with an automated workstation (Maxwell; Promega, Fitchburg, MA, USA) and checked for quality and quantity by a small scale spectrophotometer (NanoDrop; Thermo Fisher Scientific Inc., Waltham, MA, USA). Genotyping was performed using restriction fragment length polymorphism, allele-specific oligonucleotide and melting curve analysis on Applied BioSystems 7500 Real-Time PCR system (Thermo Fisher Scientific Inc.). Genotyping was performed according to the manufacturer's standard protocols. Forward and reverse primers' sequences are available upon request.

\section{Statistical Analyses}

Hardy-Weinberg Equilibrium (HWE) and Linkage Disequilibrium (LD) were tested through Haploview 3.2 software for Windows (https://www.broadinstitute.org/ haploview/haploview) [24]. Haplotypes' analysis was performed in "R" environment (http://cran.r-project.org/), using the statistics package "haplo.score".

The statistical analyses for single SNPs (ANOVA, ANCOVA, Multinomial Logistic Regression, Repeated Measures ANOVA) were performed through the use of IBM SPSS package for windows ver. 23.0 (http://www. ibm.com/analytics/us/en/technology/spss/). Genotypes and dominance/recessive models were tested. Bonferroni correction was applied to minimize false-positive risk derived from multiple testing. We took in consideration the number of SNPs tested for significance (4) in the main analysis. Significance was considered for $p<0.05 / 4=$ 0.0125 .

\section{RESULTS}

Data regarding the samples under investigation are summarized in Supplementary Table 1 (available online). Supplementary Figure 1 and Table 2 (available online) reports results of Linkage and Hardy - Weinberg Equilibrium tests on the samples under investigation. Of note, rs1116715 and rs1009750 resulted not in equilibrium in the European sample. Also, in the same sample rs1011045 showed a genotyping rate $<85 \%$ thus decreasing the power of analyses on this SNP. The genotype distribution between European and MDD Korean samples are significantly different, reflecting the overall distribution observed in larger population studies (Hapmap).

\section{Primary Outcome}

None of the analyzed SNPs either in single or in haplotypic analyses resulted associated with treatment outcomes.

\section{Secondary Data}

Analyses on MDD-KOR subsample evidenced some associations with rs7465272 and symptoms severity at baseline (HDRS.B). Correlation of some ARC haplotypes, namely rs11167152-rs7465272 and rs7465272-rs10097505, with Symptom Severity (calculated as HDRS score at Baseline) was observed in the MDD-KOR subsample. In the same sample the haplotype rs10110456-rs11167152rs7465272 (which shares two SNPs with one of the previously reported) resulted associated with symptoms improvement.

No other haplotypes resulted significant based on our analyses. Regarding exploratory analyses on other characteristics, our analyses evidenced weak associations with suicide risk and family history. In particular, rs10110456 was correlated with Family History while rs7465272 as related to suicide risk. However, none of these associations survived Bonferroni correction. No significant data was obtained on the whole KOR sample nor in the BPD-KOR subsample.

Analyses on BPD-EU sample evidenced an association of rs111667152 with symptoms severity at baseline. Also, rs7465272 was associated with body mass index (BMI). These associations did not survive Bonferroni correction.

All the details regarding the data obtained are reported in Table 1 (single SNP analyses) and Table 2 (haplotype analyses).

\section{DISCUSSION}

In this paper we focused on ARC possible influence on treatment efficacy and other clinical features in two samples of different ethnicity. The reason for this choice is based on previous research on possible associations of this gene with antidepressant efficacy (in particular with response and remission outcomes for rs10110456 and rs11167152) [13]. Unfortunately, despite the previous data, ARC variants do not seem to have any effect on treatment efficacy according to our principal data. We cannot exclude the possibility of the effect being not strong enough to be detected in our samples. ARC variants, how- 
Table 1. Overall summary of data obtained on investigated samples

\begin{tabular}{|c|c|c|c|c|c|c|}
\hline Gene & SNP & Variable $^{a}$ & Test type $^{b}$ & $p$ value & Analysis details & Confidence interval \\
\hline \multicolumn{7}{|c|}{ MDD-Korean } \\
\hline \multirow[t]{4}{*}{ ARC } & rs10110456 & Family history & $\begin{array}{l}\text { Dominant model } \\
\text { (GG vs. GA/AA) }\end{array}$ & 0.036 & $\begin{array}{c}\text { Not A vs. A: } \mathrm{B}=0.725, \mathrm{SE}=0.341 \\
p=0.033, \mathrm{OR}=2.064\end{array}$ & $1.059-4.024$ \\
\hline & rs7465272 & Suicidal ideation & $\begin{array}{l}\text { Recessive model } \\
\text { (AA vs. TA/TT) }\end{array}$ & 0.045 & $\begin{array}{l}\text { Not T vs. } \mathrm{T}: \mathrm{B}=1.023, \mathrm{SE}=0.493 \\
p=0.038, \mathrm{OR}=2.783\end{array}$ & $1.058-7.317$ \\
\hline & rs7465272 & HDRS at baseline & $\begin{array}{l}\text { Dominant model } \\
\text { (TT vs. TA/AA) }\end{array}$ & 0.027 & $\begin{array}{l}\mathrm{A} \mu=23.925, \mathrm{SE}=0.703 \\
\text { Not } \mathrm{A}=21.831, \mathrm{SE}=0.621\end{array}$ & $\begin{array}{l}22.539-25.310 \\
20.608-23.054\end{array}$ \\
\hline & & & Genotype & 0.038 & $\begin{array}{l}\mathrm{AA} \mu=25.842, \mathrm{SE}=1.659 \\
\mathrm{TA} \mu=23.506, \mathrm{SE}=0.775 \\
\text { TT } \mu=21.831, \mathrm{SE}=0.620\end{array}$ & $\begin{array}{l}22.573-29.111 \\
21.978-25.033 \\
20.609-23.053\end{array}$ \\
\hline \multicolumn{7}{|c|}{ BPD-European } \\
\hline \multirow[t]{4}{*}{ ARC } & rs7465272 & $\mathrm{BMI}$ at baseline & Genotypic & 0.020 & $\begin{array}{l}\mathrm{AA} \mu=31.087, \mathrm{SE}=2.115 \\
\mathrm{TA} \mu=25.121, \mathrm{SE}=1.052 \\
\mathrm{TT} \mu=24.534, \mathrm{SE}=1.063\end{array}$ & $\begin{array}{l}26.920-35.254 \\
23.047-27.195 \\
22.441-26.628\end{array}$ \\
\hline & & & $\begin{array}{c}\text { Recessive model } \\
\text { (AA vs. TA/TT) }\end{array}$ & 0.006 & $\begin{array}{l}\mathrm{T} \mu=24.830, \mathrm{SE}=0.746 \\
\mathrm{Not} \mathrm{T} \mu=31.087, \mathrm{SE}=2.111\end{array}$ & $\begin{array}{l}23.360-26.301 \\
26.928-35.247\end{array}$ \\
\hline & rs 11167152 & HDRS at baseline & Genotypic & 0.047 & $\begin{array}{l}\mathrm{CC} \mu=15.044, \mathrm{SE}=0.800 \\
\mathrm{GC} \mu=12.295, \mathrm{SE}=1.144 \\
\mathrm{GG} \mu=11.879, \mathrm{SE}=1.321\end{array}$ & $\begin{array}{r}13.465-16.624 \\
10.036-14.555 \\
9.270-14.488\end{array}$ \\
\hline & & & $\begin{array}{l}\text { Recessive model } \\
\text { (CC vs. GC/GG) }\end{array}$ & 0.014 & $\begin{array}{l}\mathrm{G} \mu=12.117, \mathrm{SE}=0.863 \\
\text { Not } \mathrm{G} \mu=15.044, \mathrm{SE}=0.798\end{array}$ & $\begin{array}{l}10.414-13.820 \\
13.469-16.620\end{array}$ \\
\hline
\end{tabular}

Table 1 reports the nominally significant $(p<0.05)$ data obtained from analyses on the samples under investigation.

SNP, single nucleotide polymorphism; MDD, major depressive disorder; BPD, bipolar disorder. BMI, body mass index; HDRS, Hamilton depression rating scale. $B$, unstandardized regression weight; $S E$, standard error; $O R$, odds ratio, $\mu$, mean. Both Recessive and Dominant Models were tested for each SNP under investigation. Model is defined as dominant or recessive depending on the wild type form of the SNP. Dominant model: Mutated form tested for dominant effect. Recessive model: Mutated form tested for recessive effect. Wild type definition was based on the most common allele of a SNP on European population according to PubMed SNP database.

andicates the variable tested for association. ${ }^{\mathrm{b}}$ Indicated the type of model tested for association.

Table 2. Results obtained from Haplotypic analyses on Korean-MDD sample

\begin{tabular}{|c|c|c|c|c|c|c|}
\hline Hap-freq & Hap-score & $p$ value & $\operatorname{Sim} p$ value & & Haplotypes & \\
\hline \multicolumn{7}{|c|}{ HDRS score at baseline } \\
\hline \multirow{2}{*}{ Global $=0.039$} & \multicolumn{3}{|c|}{ Max - Stat $=0.016$} & & & \\
\hline & & & & rs11167152 & rs7465272 & \\
\hline 0.731 & -248029.000 & $0.01 *$ & 0.013 & C & $\mathrm{t}$ & \\
\hline 0.256 & 249388.000 & $0.01^{*}$ & 0.012 & c & a & \\
\hline \multirow{2}{*}{\multicolumn{2}{|c|}{ Global $=0.048$}} & \multicolumn{2}{|c|}{ Max - Stat $=0.030$} & & & \\
\hline & & & & rs7465272 & rs10097505 & \\
\hline 0.486 & -161532.000 & 0.106 & 0.108 & $\mathrm{t}$ & g & \\
\hline 0.252 & -0.523 & 0.601 & 0.598 & $\mathrm{t}$ & $\mathrm{a}$ & \\
\hline 0.258 & 253307.000 & $0.01 *$ & 0.012 & a & g & \\
\hline \multicolumn{7}{|c|}{ HDRS score Improvement at baseline } \\
\hline \multirow[t]{2}{*}{ Global $=0.019$} & & \multirow{2}{*}{\multicolumn{2}{|c|}{ Max - Stat $=0.043$}} & & & \\
\hline & & & & rs10110456 & rs11167152 & rs7465272 \\
\hline 0.405 & -237403.000 & 0.02 & 0.015 & $\mathrm{a}$ & c & $\mathrm{t}$ \\
\hline 0.311 & 0.330 & 0.742 & 0.75 & g & c & $\mathrm{a}$ \\
\hline 0.284 & 222629.000 & 0.03 & 0.024 & g & c & $\mathrm{t}$ \\
\hline
\end{tabular}

MDD, major depressive disorder; Hap-freq, Haplotype frequency; Hap-score, Haplotype score; sim, simulation; SNP, single nucleotide polymorphism; HDRS, Hamilton depression rating scale.

${ }^{*} p<0.0125$.

ever, may influence symptoms severity at baseline. Interestingly, rs11167152 resulted associated with symptoms severity at baseline in both EU- (single SNP analysis) and MDD-KOR (included in a haplotypic block) samples. Hinting for a possible role of this SNP in depressive severity regardless of the diagnosis and ethnic-related differ- 
ences of the genetic background. It has to be noted, though, that data on rs11167152 in the EU-sample can only be considered as exploratory, since this SNP resulted not in $\mathrm{HW}$-equilibrium and the genotyping rates were slightly lower than the $85 \%$ threshold. According to in silico analysis of rs 11167152 through the Human Splicing Finder (HSF) prediction software (http://www.umd.be/HSF3) [25], the $G>C$ variation create a silencer consensus sequence and at the same time remove a potential enhancing site (for SF2/ASF protein). Both events may influence ARC expression. Rs11167152 also slightly alter a splice site sequence, but, according to in silico data, not enough to influence splicing. It also should be noted that the location of rs11167152 is on the 3'downstream of ARC, as such no splicing events should occur at this location likely making this alteration not influent. Likewise, rs7465272 T $>$ A variation, which as associated with symptoms severity in the KOR-only MDD subsample, potentially decreases the expression of ARC through the creation of a silencer consensus sequence. Rs 7465272 was associated with Symptoms' severity in Korean subjects but not in the European ones. However, in the latter MDD subsample it was found to be associated with body mass index. Unfortunately, we were not able to compare BMI data with the Korean one since BMI was not collected from MDD patients (only on BPD ones). From our analyses we found two sets of haplotypes seemingly associated with symptoms severity at baseline in the MDD-KOR subsample. They are rs11167152, rs7465272 and rs7465272, rs10097505. The CA and AG haplotype, respectively were both associated with an higher symptoms severity. Interestingly enough, according to in silico analyses, each of the alleles included in this haplotype are likely to cause the down regulation of ARC expression. From a functional point of view, the potential down-regulation caused by the described alterations leads to a decreased availability of the encoded protein. As such, a reduced ARC expression is likely related to a more severe symptomatology at baseline. Another haplotype within ARC, the rs10110456, rs11167152, rs7465272 GCT haplotype, resulted associated with higher improvement in the sample under analysis. In this case, the link between the evidenced effect on improvement and the biological alterations caused by alleles is less clear. Indeed, rs 11167152 C allele promote the down regulation, while rs7465272 T allele has the opposite effect (in silico predictions). The role of rs10110456 G allele is less clear. Overall, our data suggest a possible implication of lower levels of ARC with symptoms phenotype. The importance of ARC levels was described in literature: Interestingly, increased expression of Arc can be triggered by 5-HT [26] and this action may be behind 5-HT involvement in the action of antidepressant drugs [27]. Further, it was evidenced an increase of Arc mRNA levels after pharmacological treatment in specific regions of the brain (cingulate and orbital areas of the frontal cortex by $34 \%$ and $46 \%$ respectively) [27].

The simultaneous study of BPD and MDD subjects may be biased, given the apparently different nature of the two diseases. However, literature data confirm the existence of an overlapping genetic background [28]. Supported by this data, we performed our analyses in both BPD and MDD subjects in order to evidence any commonalities between the two different populations. EU subsample suffers of some limitations including HWE disequilibrium for two of the investigated SNPs, namely rs11167152 and rs1009750. Further, rs10111045 and rs11167152 genotyping rates were inferior to the $85 \%$ threshold. As such the obtained results regarding the above SNPs should be carefully interpreted, and should be considered as suggestive. The somewhat limited number of subjects involved as well as the different ethnicity may pose a limit for the detection of weak influences and replication of data due to a dissimilar genetic background, respectively. Further, EU sample was collected in a cross sectional way, therefore biases may have influenced the outcome definition.

According to our main analyses, we did not find significant associations involving ARC polymorphisms with treatment outcomes in either of the investigated samples. Thus, we did not replicate our previous findings on rs10110456 and rs11167152 ARC SNPs [13]. We did detect some data indicating ARC polymorphisms being able to influence symptoms severity at baseline in both samples. In particular, rs7465272 alone and in haplotypic combination with rs11167152 or rs10097505, showed a significant association with symptoms severity at baseline in the Korean sample. rs11167152 was also the only SNP associated with symptoms severity in the European sample. Further, haplotype rs10110456-rs11167152rs7465272 resulted associated with symptoms improvement in the Korean sample. Finally, exploratory data evidenced a possible influence of rs7465272 variants on the BMl of depressed subjects in the European sample. 
Overall, we found some weak correlations in our analyses, the most suggestive one being the rs11167152 association with symptoms severity in both samples regardless of the ethnic-related genetic differences. Unfortunately, as explained before in the limit section, rs11167152 genotyping evidenced some issues in the EU-sample. The other correlations we found were evidenced only in one or the other sample under investigation. ARC variants do not seem to modulate treatment efficacy or their effect is not strong enough to be detected in our limited sample. These variants, though, may explain a certain degree of difference on symptoms severity on subjects before treatment, especially regarding rs11167152. This hypothesized effect is likely related to ARC expression, with lower levels being associated to a more severe phenotype. Further studies, on larger samples may aid for a better evaluation of $A R C$ role in MDD severity.

\section{Acknowledgments}

All authors thank the patients for their participation in this study. This study was supported by a grant of the Korean Health Technology R\&D Project, Ministry of Health \& Welfare, Republic of Korea (HC10TISI0031). All the authors listed meet the International Committee of Medical Journal Editors (ICMJE) criteria for authorship for this work. They have given their approval for this version to be published and take responsibility for the integrity of the data reported in the work. All authors declare that they have no conflict of interest.

\section{- Conflicts of Interest}

No potential conflict of interest relevant to this article was reported.

\section{- Author Contributions}

Conceptualization: Concetta Crisafulli, Alessandro Serretti. Recruitment (EU): Laura Mandelli, Daniel Souery, Julien Mendlewicz, Alessandro Serretti. Recruitment (KOR): Soo-Jung Lee, Sheng-Min Wang, Changsu Han, Ashwin Patkar, Prakash Masand, Chi-Un Pae. Statistical Analyses: Marco Calabrò, Concetta Crisafulli, Laura Mandelli. First Draft \& Revision: Marco Calabrò, Laura Mandelli, Concetta Crisafulli, Alessandro Serretti

Supervision: Chi-Un Pae, Alessandro Serretti. All authors read and approved the final draft of the manuscript.

\section{ORCID}

Concetta Crisafulli https://orcid.org/0000-0002-9703-0083

Marco Calabrò https://orcid.org/0000-0003-2082-9855

Laura Mandelli https://orcid.org/0000-0001-9726-1802

Sheng-Min Wang https://orcid.org/0000-0002-1707-9435

Soo-Jung Lee https://orcid.org/0000-0002-1299-5266

Changsu Han https://orcid.org/0000-0002-4021-8907

Ashwin Patkar https://orcid.org/0000-0003-0484-1301

Prakash Masand https://orcid.org/0000-0003-1973-9449

Chi-Un Pae https://orcid.org/0000-0003-1632-4248

Daniel Souery https://orcid.org/0000-0003-3434-5958

Julien Mendlewicz https://orcid.org/0000-0002-6131-2732

Alessandro Serretti https://orcid.org/0000-0003-4363-3759

\section{REFERENCES}

1. Kessler RC, Aguilar-Gaxiola S, Alonso J, Chatterji S, Lee S, Ormel J, et al. The global burden of mental disorders: an update from the WHO World Mental Health (WMH) surveys. Epidemiol Psichiatr Soc 2009; 18:23-33.

2. Henter ID, de Sousa RT, Gold PW, Brunoni AR, Zarate CA Jr, Machado-Vieira R. Mood therapeutics: novel pharmacological approaches for treating depression. Expert Rev Clin Pharmacol 2017;10:153-166.

3. Serretti A. Genetics and pharmacogenetics of mood disorders. Psychiatr Pol 2017;51:197-203.

4. Mullins N, Lewis CM. Genetics of depression: progress at last. Curr Psychiatry Rep 2017;19:43.

5. Pettersson E, Lichtenstein P, Larsson H, Song J. Genetic influences on eight psychiatric disorders based on family data of 4 408646 full and half-siblings, and genetic data of 333748 cases and controls - CORRIGENDUM. Psychol Med 2019;49: 351.

6. de Jong S, Diniz MJA, Saloma A, Gadelha A, Santoro ML, Ota VK, et al. Applying polygenic risk scoring for psychiatric disorders to a large family with bipolar disorder and major depressive disorder. Commun Biol 2018;1:163.

7. Calabrò M, Mandelli L, Crisafulli C, Nicola MD, Colombo R, Janiri $\mathrm{L}$, et al. ZNF804A gene variants have a cross-diagnostic influence on psychosis and treatment improvement in mood disorders. Clin Psychopharmacol Neurosci 2020;18:231-240.

8. Calabrò M, Mandelli L, Crisafulli C, Lee SJ, Jun TY, Wang SM, et al. Genes involved in neurodevelopment, neuroplasticity and major depression: no association for CACNA1C, CHRNA7 and MAPK1. Clin Psychopharmacol Neurosci 2019;17:364-368.

9. Stahl EA, Forstner AJ, McQuillin A, Ripke S, Trubetskoy V, Mattheisen $\mathrm{M}$, et al. Genome-wide association study identifies 30 loci associated with bipolar disorder. BioRxiv 173062 [Preprint]. 2017 [cited 2019 Feb 14]. Available from: https:// doi.org/10.1101/173062. 
10. Nikolaienko O, Patil S, Eriksen MS, Bramham CR. Arc protein: a flexible hub for synaptic plasticity and cognition. Semin Cell Dev Biol 2018;77:33-42.

11. Calabrò M, Porcelli S, Crisafulli C, Wang SM, Lee SJ, Han C, et al. Genetic variants within key nodes of the cascade of antipsychotic mechanisms: effects on antipsychotic response and schizophrenia psychopathology in a naturalistic treatment setting in two independent Korean and Italian samples. Adv Ther 2017;34:1482-1497.

12. Li Y, Pehrson AL, Waller JA, Dale E, Sanchez C, Gulinello M. A critical evaluation of the activity-regulated cytoskeleton-associated protein (Arc/Arg3.1)'s putative role in regulating dendritic plasticity, cognitive processes, and mood in animal models of depression. Front Neurosci 2015;9:279.

13. Calabrò M, Fabbri C, Crisafulli C, Albani D, Forloni G, Kasper $\mathrm{S}$, et al. The serotonin transporter and the activity regulated cytoskeleton-associated protein genes in antidepressant response and resistance: 5-HTTLPR and other variants. Hum Psychopharmacol 2018;33:e2682.

14. Lyford GL, Yamagata K, Kaufmann WE, Barnes CA, Sanders LK, Copeland NG, et al. Arc, a growth factor and activityregulated gene, encodes a novel cytoskeleton-associated protein that is enriched in neuronal dendrites. Neuron 1995; 14: 433-445.

15. Messaoudi E, Kanhema T, Soulé J, Tiron A, Dagyte G, da Silva B, et al. Sustained Arc/Arg3. 1 synthesis controls long-term potentiation consolidation through regulation of local actin polymerization in the dentate gyrus in vivo. J Neurosci 2007;27: 10445-10455.

16. Fujimoto T, Tanaka H, Kumamaru E, Okamura K, Miki N. Arc interacts with microtubules/microtubule-associated protein 2 and attenuates microtubule-associated protein 2 immunoreactivity in the dendrites. J Neurosci Res 2004;76:51-63.

17. Balu DT, Coyle JT. Chronic D-serine reverses arc expression and partially rescues dendritic abnormalities in a mouse model of NMDA receptor hypofunction. Neurochem Int 2014;75: 76-78.

18. Peebles CL, Yoo J, Thwin MT, Palop JJ, Noebels JL, Finkbeiner S. Arc regulates spine morphology and maintains network stability in vivo. Proc Natl Acad Sci U S A 2010;107:1817318178.

19. Souery D, Zaninotto L, Calati R, Linotte S, Mendlewicz J, Sentissi $\mathrm{O}$, et al. Depression across mood disorders: review and analysis in a clinical sample. Compr Psychiatry 2012;53: 24-38.

20. Sheehan DV, Lecrubier $Y$, Sheehan KH, Amorim P, Janavs J, Weiller E, et al. The Mini-International Neuropsychiatric Interview (M.I.N.I.): the development and validation of a structured diagnostic psychiatric interview for DSM-IV and ICD-10. J Clin Psychiatry 1998;59 Suppl 20:22-33;quiz 34-57.

21. Calabrò M, Mandelli L, Crisafulli C, Sidoti A, Jun TY, Lee SJ, et al. Genes involved in neurodevelopment, neuroplasticity, and bipolar disorder: CACNA1C, CHRNA1, and MAPK1. Neuropsychobiology 2016;74:159-168.

22. Mandelli L, Wang SM, Han C, Lee SJ, Patkar AA, Masand PS, et al. The impact of a single nucleotide polymorphism in SIGMAR1 on depressive symptoms in major depressive disorder and bipolar disorder. Adv Ther 2017;34:713-724.

23. Schosser A, Serretti A, Souery D, Mendlewicz J, Zohar J, Montgomery S, et al. European Group for the Study of Resistant Depression (GSRD)--where have we gone so far: review of clinical and genetic findings. Eur Neuropsychopharmacol 2012;22:453-468.

24. Barrett JC, Fry B, Maller J, Daly MJ. Haploview: analysis and visualization of $L D$ and haplotype maps. Bioinformatics 2005;21:263-265.

25. Desmet FO, Hamroun D, Lalande M, Collod-Béroud G, Claustres M, Béroud C. Human Splicing Finder: an online bioinformatics tool to predict splicing signals. Nucleic Acids Res 2009;37:e67.

26. Pei Q, Lewis L, Sprakes ME, Jones EJ, Grahame-Smith DG, Zetterström TS. Serotonergic regulation of $m R N A$ expression of Arc, an immediate early gene selectively localized at neuronal dendrites. Neuropharmacology 2000;39:463-470.

27. De Foubert G, Carney SL, Robinson CS, Destexhe EJ, Tomlinson R, Hicks CA, et al. Fluoxetine-induced change in rat brain expression of brain-derived neurotrophic factor varies depending on length of treatment. Neuroscience 2004; 128:597-604.

28. Coleman JRI, Gaspar HA, Bryois J, Byrne EM, Forstner AJ, Holmans PA, et al. The genetics of the mood disorder spectrum: genome-wide association analyses of over 185,000 cases and 439,000 controls. BioRxiv 383331 [Preprint]. 2019 [cited 2019 Feb 14]. Available from: https://doi.org/10.1101/ 383331. 